\section{Adaptação transcultural de escalas de aderência ao tratamento em hemodiálise: Renal Adherence Behaviour Questionnaire (RABQ) e Renal Adherence Attitudes Questionnaire (RAAQ)}

\author{
Transcultural adaptation of scales for treatment \\ adherence in hemodialysis: Renal Adherence \\ Behaviour Questionnaire (RABQ) and Renal \\ Adherence Attitudes Questionnaire (RAAQ)
}

\author{
Adaptación transcultural de las escalas de \\ adherencia al tratamiento de hemodiálisis: Renal \\ Adherence Behaviour Questionnaire (RABQ) y \\ Renal Adherence Attitudes Questionnaire (RAAQ)
}

Inês Maria de Jesus Machado 1 Marina Bittencourt Bandeira 1 Hélady Sanders Pinheiro 2 Nathália dos Santos Dutra 1

\footnotetext{
1 Universidade Federal de São João del-Rei, São João Del Rei, Brasil.

2 Universidade Federal de Juiz de Fora, Juiz de Fora Brasil.

Correspondência I. M. J. Machado Rua Delegado José Lima 109, apto. 105, São João Del Rei, MG 36302-022, Brasil. inesmjmachado@yahoo. com.br
}

\begin{abstract}
Treatment adherence in hemodialysis is important for guaranteeing better results for patients, but Brazil still lacks validated assessment tools for this purpose. The current study aimed to perform a cross-cultural adaptation of the Renal Adherence Behaviour Questionnaire (RABQ) and the Renal Adherence Attitudes Questionnaire (RAAQ). The two questionnaires were sub mitted to the following cross-cultural adaptation procedures: translation, back-translation, expert panel review, and pilot study. Changes were made in the items' wording and application, which requires a face-to-face interview. It was not necessary to change the choices of an swers. The Brazilian versions of the RABQ and $R A A Q$ showed semantic and cultural equivalence to the original versions and are easy for the target population to understand. The two scales still require validity and reliability studies before use in the field.
\end{abstract}

Medication Adherence; Renal Dialysis; Questionnaires

\section{Resumo}

A aderência dos pacientes ao tratamento em hemodiálise é importante para o sucesso do tratamento, mas há carência de instrumentos de avaliação validados para o Brasil. Esta pesquisa visou à adaptação transcultural para o Brasil das escalas Renal Adherence Behaviour Questionnaire (RABQ) $e$ Renal Adherence Attitudes Questionnaire (RAAQ), que avaliam os comportamentos e atitudes de aderência. Esses instrumentos foram submetidos aos procedimentos de adaptação transcultural: tradução, retradução, avaliação por comissão de especialistas e estudo piloto. Foram feitas modificações na redação dos itens e no formato de aplicação, que deve ser em entrevista face a face. Não foi necessário alterar as alternativas de respostas. As versões brasileiras das escalas RABQ e RAAQ apresentam equivalência semântica e cultural com as versões originais e foram redigidas de forma a facilitar sua compreensão pela população-alvo. As duas escalas necessitam ser submetidas a estudos de validade e fidedignidade para serem utilizadas.

Adesão à Medicação; Diálise Renal; Questionários 


\section{Introdução}

A doença renal crônica é considerada um grande problema de saúde pública, causando elevadas taxas de morbidade e mortalidade. Em 2012, o Brasil possuía 696 centros de diálise cadastrados no país e 97.586 pacientes em diálise distribuídos por todos os estados 1 .

A doença renal crônica é caracterizada pela presença de lesão renal ou diminuição da função dos rins por três meses ou mais 2 . $\mathrm{O}$ tratamento hemodialítico requer aderência medicamentosa, dietética, hídrica e de assiduidade às sessões de diálise ${ }^{3}$. A falta de aderência ao tratamento pode ser a causa do insucesso das propostas terapêuticas, colaborando para o aumento da morbidade e da mortalidade 4 .

Segundo a Organização Mundial da Saúde (OMS), os provedores e o sistema de saúde precisam desenvolver meios de avaliar a aderência e os seus fatores associados, objetivando melhorar as intervenções terapêuticas 5. Dessa forma, um dos instrumentos para avaliar a aderência ao tratamento em hemodiálise, com qualidades psicométricas adequadas, citado na literatura internacional, consiste nas escalas desenvolvidas por Rushe \& McGee ${ }^{6}$ denominadas Renal Adherence Attitudes Questionnaire (RAAQ) e Renal Adherence Behaviour Questionnaire (RABQ).

No Brasil, há uma carência de escalas de medida para avaliar especificamente a aderência ao tratamento em hemodiálise. O objetivo do presente trabalho foi fazer a adaptação transcultural das escalas RAAQ e RABQ para o Brasil.

\section{Método}

\section{Seleção da amostra}

Foi selecionada uma amostra de conveniência de 21 pacientes com doença renal crônica em uma clínica de hemodiálise. Os critérios de inclusão foram: ter diagnóstico confirmado de doença renal crônica, estar em tratamento há mais de um ano e ter idade igual ou superior a 18 anos. $\mathrm{O}$ tamanho da amostra é compatível com estudos que visam testar a facilidade de compreensão dos itens pela população-alvo 7. Não houve necessidade de excluir qualquer paciente.

\section{Instrumentos de medida}

A escala RAAQ possui 26 itens que avaliam as atitudes dos pacientes em relação ao tratamento mediante uma estrutura de quatro fatores: (1) atitudes frente às restrições sociais; (2) atitudes em relação ao bem-estar; (3) atitudes em relação ao autocuidado e suporte familiar; e (4) aceitação. As opções de resposta variam entre concordo totalmente e discordo totalmente. A maior pontuação na escala está associada a uma atitude mais positiva em relação às restrições alimentares e hídricas.

A escala RABQ possui 25 itens que avaliam o comportamento dos pacientes frente às restrições de potássio, fósforo, sódio e líquido e à tomada de medicamento mediante uma estrutura de cinco fatores: (1) aderência às restrições de líquidos; (2) aderência às restrições de potássio e fósforo; (3) aderência ao autocuidado; (4) aderência nos momentos de dificuldade; e (5) aderência em relação à ingestão de sódio. As opções de resposta variam entre nunca e sempre. Os maiores escores nessa escala indicam maior frequência de comportamentos de aderência.

\section{Procedimento de adaptação transcultural}

Após a autorização dos autores das escalas, o projeto foi submetido e aprovado pela Comissão de Ética da Universidade Federal de São João del-Rei - UFSJ (protocolo no 005/2012). Foram realizados os procedimentos descritos abaixo, recomendados pela literatura internacional da área 7,8 .

a) Tradução: esta etapa consistiu na realização independente de duas traduções dos instrumentos originais por duas pessoas bilíngues, cuja língua materna era o português (T1 e T2).

b) Retradução: as duas traduções foram retraduzidas para o inglês, resultando em duas retraduções (R1 e R2). Essas retraduções foram feitas, de forma independente, por duas outras pessoas bilíngues cuja língua materna era o inglês.

c) Análise por comissão de especialistas: as versões das escalas foram analisadas por uma comissão de especialistas, composta por um especialista em linguística e duas psicólogas. Foi realizada uma comparação entre as versões brasileiras e as originais, visando identificar possíveis imprecisões e realizar as suas correções, de forma a garantir a equivalência semântica das versões em português, em relação à escala original. Foram feitas também modificações na redação dos itens, objetivando adaptá-los à cultura brasileira. Foram feitas duas avaliações: a primeira (A1) se referiu à porcentagem de concordância entre os significados das versões traduzidas e originais. A segunda avaliação (A2) indicou o grau de alteração observado (pouco alterado, inalterado, muito alterado e completamente alterado). Foi obtida, nesta fase, uma versão preliminar das duas escalas. 
A versão preliminar foi submetida à apreciação de uma comissão composta por profissionais de saúde de uma clínica de hemodiálise. As sugestões desses profissionais foram submetidas à comissão de especialistas que deliberou sobre sua pertinência.

d) Estudo piloto: esta etapa consistiu na avaliação da compreensão e aplicabilidade das escalas junto à população-alvo. Foi utilizada a Técnica de Sondagem ("probe technique"), na qual se solicita que os respondentes expliquem o que entenderam de cada item e sugiram modificações, quando necessário ${ }^{7}$. Obteve-se assim a versão final das duas escalas adaptadas para o contexto brasileiro

\section{Resultados}

\section{Descrição da amostra}

Os participantes tinham a média de idade de 53 anos, sendo a maioria do sexo feminino $(52,4 \%)$, casado $(81 \%)$, com 4 a 8 anos de escolaridade $(66,7 \%)$ e renda entre 1 a 2 salários mínimos $(61,9 \%)$. A maioria $(76,2 \%)$ estava em hemodiálise por cinco anos ou mais. O diagnóstico de base mais frequente, representado por pouco mais de um terço, foi a nefroesclerose hipertensiva $(38,09 \%)$.

\section{Resultados da adaptação transcultural}

A Tabela 1 apresenta os resultados da adaptação transcultural das duas escalas. A primeira coluna de cada escala apresenta os itens da versão original em inglês e a segunda coluna, a versão final em português, após as modificações realizadas pela comissão de especialistas, com base na análise das traduções e retraduções, nas sugestões dadas pelos profissionais de saúde da clínica de hemodiálise e nas informações obtidas após o estudo piloto com a população-alvo.

Para a escala RAAQ, 24 dos 26 itens obtiveram percentual de concordância entre as versões acima de 90\%, na avaliação A1. Para a avaliação A2, a maioria (20 itens) foi classificada como pouco alterados ou inalterados; três itens foram pouco alterados, completamente alterados e muito alterados, respectivamente, e o restante (3 itens) não obteve nenhuma alteração. As alternativas de resposta tiveram $100 \%$ de concordância, resultando em: concordo totalmente, concordo, indeciso, discordo, discordo totalmente.

Tabela 1

Resultados do processo de adaptação transcultural das escalas Renal Adherence Attitudes Questionnaire (RAAQ) e Renal Adherence Behaviour Questionnaire (RABQ).

\begin{tabular}{|c|c|c|c|}
\hline \multicolumn{2}{|c|}{ Escala RAAQ } & \multicolumn{2}{|c|}{ Escala RABQ } \\
\hline Versão original & Versão final & Versão original & Versão final \\
\hline $\begin{array}{l}\text { 1. My diet fits easily into my } \\
\text { present lifestyle. }\end{array}$ & $\begin{array}{c}\text { 1. Minha dieta se encaixa (ajusta) } \\
\text { facilmente em meu estilo de vida } \\
\text { atual. }\end{array}$ & $\begin{array}{l}\text { 1. I listen to the advice of my } \\
\text { dietician. }\end{array}$ & $\begin{array}{l}\text { 1. Eu escuto os conselhos da minha } \\
\text { nutricionista. }\end{array}$ \\
\hline $\begin{array}{l}\text { 2. Patients on dialysis should be } \\
\text { able to make up their own minds } \\
\text { what to eat. }\end{array}$ & $\begin{array}{l}\text { 2. Pacientes em diálise poderiam } \\
\text { decidir por eles mesmos o que } \\
\text { comer. }\end{array}$ & $\begin{array}{c}\text { 2. I have difficulty restricting my } \\
\text { intake of beer/wine. }\end{array}$ & $\begin{array}{l}\text { 2. Eu tenho dificuldades de controlar } \\
\text { meu consumo de bebidas alcoólicas. }\end{array}$ \\
\hline $\begin{array}{l}\text { 3. Restricting my intake of fluid is } \\
\text { vital for my physical well-being. }\end{array}$ & $\begin{array}{l}\text { 3. Controlar meu consumo de } \\
\text { líquidos é essencial para meu bem- } \\
\text { estar físico. }\end{array}$ & $\begin{array}{l}\text { 3. There are times when I cannot } \\
\text { resist forbidden foods. }\end{array}$ & $\begin{array}{l}\text { 3. Às vezes não consigo resistir a } \\
\text { comidas proibidas. }\end{array}$ \\
\hline $\begin{array}{l}\text { 4. Having to adhere to such a strict } \\
\text { diet prevents me from attending } \\
\text { social functions. }\end{array}$ & $\begin{array}{l}\text { 4. Ter que aderir a uma dieta rígida } \\
\text { me impede de participar de festas. }\end{array}$ & 4. I bargain over food. & $\begin{array}{l}\text { 4. Eu negocio (troco/barganho) } \\
\text { minha dieta de alimentos. }\end{array}$ \\
\hline $\begin{array}{l}\text { 5. I feel that the dietician } \\
\text { places too much importance on } \\
\text { restricting fluid. }\end{array}$ & $\begin{array}{l}\text { 5. Eu sinto que o nutricionista dá } \\
\text { importância demais ao controle de } \\
\text { líquidos. }\end{array}$ & $\begin{array}{l}\text { 5. Breaking my diet makes no } \\
\text { difference to my health. }\end{array}$ & $\begin{array}{l}\text { 5. Quebrar minha dieta não faz } \\
\text { diferença para minha saúde. }\end{array}$ \\
\hline $\begin{array}{l}\text { 6. I feel the benefits of restricting } \\
\text { my intake of foods, e.g. fruit and } \\
\text { vegetables. }\end{array}$ & $\begin{array}{l}\text { 6. Eu sinto as vantagens de controlar } \\
\text { minha ingestão de alimentos. }\end{array}$ & $\begin{array}{l}\text { 6. I do not care what I eat when I am } \\
\text { feeling upset. }\end{array}$ & $\begin{array}{l}\text { 6. Não me importo com o que } \\
\text { como quando estou me sentindo } \\
\text { chateado. }\end{array}$ \\
\hline
\end{tabular}

(continua) 


\begin{tabular}{|c|c|}
\hline \multicolumn{2}{|c|}{ Escala RAAQ } \\
\hline Versão original & Versão final \\
\hline $\begin{array}{l}\text { 7. I am able to drink as much fluid } \\
\text { today as I used to. }\end{array}$ & $\begin{array}{l}\text { 7. Eu posso beber tanto líquido hoje } \\
\text { quanto antes. }\end{array}$ \\
\hline $\begin{array}{l}\text { 8. I worry a lot about having } \\
\text { gained excessive weight (e.g. over } \\
\text { 4lb./2kg) between dialyses. }\end{array}$ & $\begin{array}{l}\text { 8. Eu me preocupo muito quando } \\
\text { ganho peso demais entre diálises. }\end{array}$ \\
\hline $\begin{array}{l}\text { 9. I feel the dietician places too } \\
\text { much importance on restricting } \\
\text { certain foods. }\end{array}$ & $\begin{array}{l}\text { 9. Eu sinto que o nutricionista coloca } \\
\text { muita importância em controlar } \\
\text { certos alimentos. }\end{array}$ \\
\hline $\begin{array}{l}\text { 10. As time goes by, I find it easier } \\
\text { to keep to my diet. }\end{array}$ & $\begin{array}{l}\text { 10. Com o passar do tempo eu acho } \\
\text { mais fácil manter minha dieta. }\end{array}$ \\
\hline $\begin{array}{l}\text { 11. I do not usually feel the } \\
\text { benefits of keeping to such a strict } \\
\text { diet. }\end{array}$ & $\begin{array}{l}\text { 11. Geralmente eu não sinto as } \\
\text { vantagens de manter uma dieta tão } \\
\text { controlada. }\end{array}$ \\
\hline $\begin{array}{l}\text { 12. It does not worry me if I gain } \\
\text { weight between dialysis sessions. }\end{array}$ & $\begin{array}{c}\text { 12. Eu não me preocupo (importo) } \\
\text { se ganho peso entre as sessões de } \\
\text { diálises. }\end{array}$ \\
\hline $\begin{array}{l}\text { 13. I feel the benefits of restricting } \\
\text { salt. }\end{array}$ & $\begin{array}{l}\text { 13. Sinto as vantagens do controle } \\
\text { de sal. }\end{array}$ \\
\hline $\begin{array}{l}\text { 14. I feel better when I keep to the } \\
\text { prescribed diet. }\end{array}$ & $\begin{array}{l}\text { 14. Eu me sinto melhor quando } \\
\text { mantenho a dieta indicada. }\end{array}$ \\
\hline
\end{tabular}

\section{Having to restrict fluid} prevents me from enjoying myself. 16. It is often too much trouble to eat different foods to the rest of my family.

17. My diet severely disrupts my life.

18. My diet is costly to maintain. 19. I do not allow my dietary restrictions to prevent me from attending social functions. 20. My family and friends are important in helping me keep to the diet.

21. Breaking my diet does not have any consequences to my health.

22. I feel I am unable to accept the restrictions to my life as a result of dialysis.

23. As time goes by I find it more difficult to keep to my diet.

24. I feel guilty when I break my diet.

25. I find my diet fits easily into my life.

26. I become frustrated trying to keep to such a strict diet.

Escala RABQ

Versão original

Versão final

7. I drink as much fluid today as I

15. Ter de controlar líquidos impede que eu me divirta.

16. Muitas vezes, dá muito trabalho comer alimentos diferentes do resto da minha família.

17. Minha dieta atrapalha demais minha vida.

18. É caro manter minha dieta.

19. Eu não permito que minhas limitações alimentares me impeçam de participar de festas.

20. Minha família e amigos são importantes para me ajudar a manter

$$
\text { a dieta. }
$$

21. Quebrar minha dieta não traz nenhuma consequência a minha saúde.

22. Eu sinto que não sou capaz de aceitar limitações para minha vida por causa da diálise.

23. Com o passar do tempo, eu acho 23. Overall, I restrict my salt intake. mais difícil seguir minha dieta.

24. Sinto-me culpado quando não sigo minha dieta.

25. Eu acho que a minha dieta se encaixa (ajusta) facilmente em minha vida.

26. Fico frustrado em manter uma dieta tão rigorosa. ever did.

8. My family help me keep to my diet.

9. I do not keep to my diet when eating in restaurants.

10. I drink more than the recommended fluid allowance when I am upset.

11. I avoid foods with a high salt content.

12. I am careful to keep to the prescribed fluid allowance.

13. I drink at least 5-6 mugs of fluid daily.

14. I am sometimes preoccupied with food which I am not supposed to eat.

7. Eu bebo tanto líquido atualmente como sempre fiz.

8. Minha família me ajuda a manter a minha dieta.

9. Não faço a minha dieta quando como fora.

10. Eu bebo mais líquido do que o recomendado quando estou chateado.

11. Eu evito comer alimentos com muito sal.

12. Eu tomo cuidado em manter a quantidade de líquidos indicada.

13. Bebo pelo menos 5-6 copos de líquidos por dia.

14. Às vezes fico preocupado com alimentos que eu não devo comer.

15. I take my medication as prescribed.

16. I am careful to weigh food before eating it.

17. It is difficult for me to restrict fluid during the summer.

18. I weigh myself regularly.

19. I get away with drinking more than the recommended amount of fluid.

20. I decide what I eat, even if it goes against the advice of the dietician.

21. I use salt at mealtimes.

15. Eu tomo minha medicação da forma indicada.

16. Eu avalio cuidadosamente a quantidade de alimentos antes de comê-los.

17. É difícil, para mim, controlar líquidos durante o verão.

18. Eu me peso regularmente.

19. Eu consigo beber mais do que a quantidade recomendada de líquidos.

20. Eu decido o que comer, mesmo que seja contra o conselho do nutricionista.

21. Eu uso sal na hora das refeições.

22. Overall, I restrict my potassium intake.

22. Em geral, eu controlo meu consumo de alimentos que têm potássio.

23. Em geral, eu controlo meu consumo de sal.

24. Overall, I take my medication.

25. Overall, I keep to the recommended fluid intake.
24. Eu geral, eu tomo minha medicação.

25. Em geral, eu sigo as recomendações de líquidos. 
Para a escala RABQ, 23 dos 25 itens obtiveram porcentagens acima de $88 \%$ de concordância entre as versões, na avaliação A1. Foram também considerados, em sua maioria (90\%), como pouco alterados ou inalterados, na avaliação A2. As alternativas de resposta obtiveram alto nível de concordância, resultando nos termos: nunca, raramente, às vezes, frequentemente e sempre.

Os resultados do estudo piloto indicaram que os itens das duas escalas foram de fácil compreensão para os pacientes. Apenas sete itens da escala RAAQ e seis da escala RABQ necessitaram de pequenas modificações. Não foi necessário alterar as alternativas de respostas. A comissão de especialistas sugeriu uma mudança no formato de aplicação das escalas, de autorrelato para a aplicação em entrevista face a face.

\section{Discussão}

Este estudo permitiu realizar a adaptação transcultural para o Brasil das escalas RAAQ e RABQ de avaliação da aderência ao tratamento em hemodiálise. A fase de análise das versões das escalas pela comissão de especialistas possibilitou corrigir imprecisões nas traduções e ajustar a redação dos itens ao contexto cultural brasileiro. As sugestões dos profissionais da clínica de hemodiálise e o estudo piloto colaboraram para a obtenção de escalas adaptadas à população-alvo de pacientes de qualquer nível de escolaridade, conforme recomendação de Pasquali 9 . A técnica de sondagem foi essencial para evitar dificulda- des de compreensão dos itens que podem afetar a fidedignidade das escalas 7 .

Os procedimentos de adaptação transcultural adotados serviram para garantir a equivalência semântica e cultural das versões brasileiras em relação às escalas originais, conforme recomendações de Guillemin et al. 7 e Beaton et al. ${ }^{8}$.

O modo de aplicação das escalas, em formato de entrevista face a face e não autoadministradas é considerado mais adequado para a populaçãoalvo com baixa escolaridade ${ }^{9}$, tendo em vista a sua dificuldade de leitura e compreensão das questões e de marcação de opções de resposta, que poderiam invalidar os dados. Quanto à probabilidade de respostas falsas, as duas formas podem igualmente sofrer influência do desejo social. O uso da técnica de sondagem em entrevistas de coleta de dados pode controlar tal viés. A amostra estudada, nesta pesquisa, apresentou características sociodemográficas e clínicas semelhantes às dos estudos de validação das escalas originais 6 , e da população de pacientes que usualmente fazem tratamento em hemodiálise no contexto brasileiro ${ }^{1}$.

Espera-se que essas escalas possam ser úteis, no contexto clínico de hemodiálise, para a elaboração de planos terapêuticos individualizados e, no contexto da pesquisa, para a avaliação dos fatores determinantes da não aderência. Todavia, estudos futuros deverão avaliar as qualidades psicométricas de validade e fidedignidade das escalas, para que elas possam ser utilizadas no contexto brasileiro. 


\section{Resumen}

La adherencia de los pacientes al tratamiento en hemodiálisis es fundamental para un tratamiento exitoso, pero hay una falta de instrumentos de evaluación validados para Brasil. Esta investigación tuvo como objetivo realizar la adaptación transcultural para Brasil de las escalas: Renal Adherencia Behaviour Questionnaire $(R A B Q)$ y Renal Adherence Attitudes Questionnaire (RAAQ), que evalúan los comportamientos y actitudes de adherencia. Estos instrumentos fueron sometidos a procedimientos de adaptación transcultural: traducción, retrotraducción, revisión por una comisión de expertos y estudio piloto. Se realizaron cambios en la redacción de los artículos y el formato de aplicaciones, que deben ser mediante entrevista. No fue necesario modificar las opciones de respuesta. Las versiones brasileñas de las escalas tienen equivalencia semántica y cultural con las versiones originales y se redactaron para facilitar su comprensión por parte de la población y de los pacientes. Las dos escalas deben ser objeto de estudios de validez y fiabilidad para su uso.

Cumplimiento de la Medicación; Diálisis Renal; Cuestionarios

\section{Colaboradores}

I. M. J. Machado realizou a coleta, analise de dados, redação e aprovação do artigo. M. B. Bandeira orientou a pesquisa e a redação e revisão do artigo. H. S. Pinheiro participou como coorientadora da pesquisa e colaborou na redação e revisão do artigo. N. S. Dutra colaborou na revisão de literatura, coleta dos dados, redação e revisão do manuscrito.

\section{Referências}

1. Sesso R, Lopes AA, Thomé FS, Lugon JR, Watanabe Y, Santos DR. Relatório do censo brasileiro de diálise crônica de 2012. J Bras Nefrol 2014; 36:48-5.

2. National Kidney Foundation. K/DOQI clinical practice guidelines for chronic kidney disease: evaluation, classification, and stratification. Am J Kidney Dis 2002; 39(2 Suppl 1):S1-266.

3. Riella MC. Princípios de nefrologia e distúrbios hidroeletrolíticos. Rio de Janeiro: Editora Guanabara Koogan; 2003.

4. Green CA. What can patient health education coordinators learn from ten years of compliance research? Patient Educ Couns 1987; 10:167-74.

5. Word Health Organization. Adherence to long term therapies: evidence for action. Geneva: World Health Organization; 2003.

6. Rushe H, McGee HM. Assessing adherence to dietary recommendations for hemodialysis patients: the Renal Adherence Attitudes Questionnaire (RAAQ) and the Renal Adherence Behaviour Questionnaire (RABQ). J Psychosom Res 1998; 45:149-57.

7. Guillemin F, Bombardier C, Beaton D. Cross-cultural adaptation of health-related quality of life measures: literature review and proposed guidelines. J Clin Epidemiol 1993; 46:1417-32.

8. Beaton DE, Bombardier CMD, Guillemin FMD, Ferraz MB. Guidelines for the process of crosscultural adaptation of self-report measures. Spine (Phila Pa 1976) 2000; 25:3186-91.

9. Pasquali L. Princípios de elaboração de escalas psicológicas. In: Gorenstein C, Andrade LHSG, Zuardi AW, organizadores. Escalas de avaliação clínica em psiquiatria e psicofarmacologia. São Paulo: Editora Lemos; 2000. p. 15-21.

Recebido em 25/Jun/2014

Versão final reapresentada em 29/Mai/2015 Aprovado em 27/Ago/2015 